\title{
SavingLife: An Educational Technology for Basic and Advanced Cardiovascular Life Support
}

\author{
Najma Naz ${ }^{1, *(1)}$, Ali Sayyed ${ }^{2}\left(\mathbb{D}\right.$, Grace Teresinha Marcon Dal Sasso ${ }^{3}$, Sabiha Khanum ${ }^{4}$ (i) and \\ Maria de Lourdes de Souza ${ }^{3}$ \\ 1 Postgraduate College of Nursing (PGCN), Phase-V, Hayatabad, Peshawar 25000, Pakistan \\ 2 Department of Computer Science, CECOS University of IT and Emerging Sciences, Phase-VI, Hayatabad, \\ Peshawar 25000, Pakistan; ali.sayyed@hotmail.com \\ 3 Postgraduate Program in Nursing (PEN), Federal University of Santa Catarina, Florianópolis 88040-900, \\ Brazil; gracetmds@gmail.com (G.T.M.D.S.); repensul@uol.com.br (M.d.L.d.S.) \\ 4 Farkhanda Institute of Nursing \& Public Health, Gandhara University, Nasir Bagh Road, Peshawar 25000, \\ Pakistan; sabiha.khanum@hotmail.com \\ * Correspondence: najma.sayyed@live.com; Tel.: +92-313-940-1493
}

Received: 14 April 2018; Accepted: 24 May 2018; Published: 1 June 2018

\begin{abstract}
The development of information and communication technologies and the accessibility of mobile devices has increased the possibilities of the teaching and learning process anywhere and anytime. Mobile and web application allows the production of constructive teaching and learning models in various educational settings, showing the potential for active learning in health. The objective of this study was to present the design and development of an educational technology (SavingLife, a web, and mobile-based application) for learning cardiopulmonary resuscitation and advanced cardiovascular life support for adults. SavingLife is a technological production, based on the concept of virtual learning and problem-based learning approaches. SavingLife was developed using five phases (analyze, design, develop, implement, evaluate) of the instructional systems development process. The technology presents 10 scenarios and 12 simulations, covering different aspects of basic and advanced cardiac life support skills. The contents can be accessed in a non-linear way leaving the students free to build their knowledge based on their previous experience. Each scenario is presented through interactive tools such as scenario description, assessment, diagnose, intervention and re-evaluation. Animated ECG rhythms, text documents, images, and videos are provided to support procedural and active learning considering the real-life situations. Accessible equally on small to large devices with or without an internet connection, SavingLife offers a dynamic, interactive and flexible tool, placing students at the center of the learning process. SavingLife can contribute to the student's learning in the assessment and management of basic and advanced cardiac life support for adults, in a safe and ethical way.
\end{abstract}

Keywords: Problem-Based Learning; cardiopulmonary resuscitation; health education; basic cardiac life support; advanced cardiac life support; educational technology

\section{Introduction}

Cardiac arrest occurs when the heart stops pumping adequate blood to the brain and body. This may be due to abnormal or irregular heart rhythm, also known as Arrhythmia. Cardiac arrest is reversible, if treated with immediate defibrillation if a shockable rhythm exist otherwise cardiopulmonary resuscitation (CPR) is used in order to induce a shockable rhythm [1,2].

The cardiovascular disease strikes every nation around the world. Despite recent advances in the prevention and treatment, cardiac arrest remains a significant public health problem and a 
leading cause of death in many parts of the world [3]. About 17.5 million people die each year from cardiovascular diseases (CVDs), which represents about $31 \%$ of all deaths globally [4].

More than $75 \%$ of all CVD deaths occur in low and middle-income countries while $80 \%$ of all CVDs deaths are due to heart attacks and strokes [4]. The estimated incidence of out-of-hospital cardiac arrest in the US and Canada is about 50 to 55/100,000 persons/year and approximately 25\% of these have pulseless ventricular arrhythmias [5]. The estimated incidence of in-hospital cardiac arrest is 3 to $6 / 1000$ admissions and similarly, approximately $25 \%$ of these have pulseless ventricular arrhythmias [6]. The vast majority of cardiac arrest victims are adults, but thousands of infants and children suffer either an in-hospital or out-of-hospital cardiac arrest each year in the US and Canada [6]. Cardiac arrest continues to be a common cause of premature death, and small improvements in survival can translate into thousands of lives saved every year. In Brazil about 347/100,000 person/year die due to cardiovascular diseases (CVD) and 134/100,000 by chronic heart diseases (CHD) [7].

These deaths can be often prevented by timely and effective lifesaving interventions, such as cardiopulmonary resuscitation (CPR) and Automated External Defibrillator (AED) if a shockable rhythm is present. Recognition of a cardiac arrest and prompt action by the rescuer are vital for the survival of victim $[8,9]$.

Nurses are usually the first responder to witness cardiac arrests in hospitals, clinics, and other health facilities [10]. They are working in the departments such as cardiology and ICU are the ones who frequently call the emergency staff. Nurses and other health professionals must have updated technical knowledge, decision-making abilities, and practical skills in order to contribute more efficiently to cardiac arrest cases. However, it is reported that most of the nurses do not feel confident while performing CPR and ACLS in emergency situations, especially in Cardiopulmonary Arrest (CPA) cases [11]. Resuscitation attempts are not always appropriate and as a result, many lives are lost [8].

Conventional training methods are often insufficient to train professionals to deliver high-quality CPR and ACLS. In an instructor-led Learning, all contents are provided in a face-to-face manner in which the teachers talk, and the students listen during $70 \%$ of class time. This method can introduce new ideas and transmit knowledge but it is not effective because students have to study intensively over a short time period. With traditional education, students may become incapable of professional judgment and critical thinking in case of the variable clinical environment [12].

More effective learner-centered education techniques are required since educators can no longer rely on traditional teaching methods. Studies indicated that the old ACLS training program has proven inconsistent and inadequate, with numerous studies reporting that trainees have poor retention and ineffective ACLS skills [13].

Problem-Based Learning (PBL), on the other hand, has been indicated an effective approach for training nurses and other health professionals, resulting in a better integration of the theory and practice $[11,14]$. Its effective learning approach prepares students as critical thinkers and able them to respond well in different health settings. PBL encourages students to take a more active role in their learning process and allow them to start building a solid knowledge base.

Information and communication technologies (ICT) are rapidly improving and easily available. The number of mobile phones in the world was 97 per 100 people at the end of 2014 [15] and has exceeded the world population in 2016 and among those more than 50\% are smartphones [16]. Similarly the number of internet users worldwide was 3 billion in 2015 [15,17]. Mobile and wireless technologies offer exciting opportunities for a low cost, high reach educational and health care services for masses [18].

Mobile phones are in the hands of increasingly more people including those in some of the poorest and most difficult to reach areas of the world [19]. Mobile phone and internet connectivity are creating unprecedented opportunities by facilitating interactions and information sharing between people [20]. In recent years, the development and applicability of informatics have influenced all areas of knowledge, disseminating information, creating educational programs, and enabling new forms of learning. 
Web and computer-based interactive and self-learning programs to train people using a micro-simulation are effective in BLS and ACLS skills [21]. Web and computer-based programs include theory, training (with or without scenarios) and self-testing [21]. In a web-based application, students are provided with an account to use the online content. Teacher-to-student and student-to-student interaction occur through asynchronous communication. The application consists of sections covering the theory, text, videos, a section for self-assessment, and sections where the trainee can simulate each scenario if any.

Various methods, including self-instruction, web-based programs, and DVD-based training, have been used to try to improve the acquisition of basic and Advanced Life Support skills. However, the survival rate from cardiac arrest remains poor despite advances in CPR training and therapies while it is a fact that the quality of CPR influences the outcome of cardiac arrest patients [9].

With web-based interactive programs it might be possible to achieve proficiency in BLS and ACLS skills; however, in order to make use of its full advantages, integration of PBL approach, and structuring the contents in the form of clinical scenarios near to real life situations, is very important [21-23].

Web-based applications open the possibility of mass public training via the Internet but for better results, the content should focus on scenario-based training and instructor's feedback for answering student questions related with the emergency conditions should be available to learners [23].

The aim of this work was to design and structure the content of basic and advanced life support for adults using the updated 2015 American Heart Association guidelines that meet both the demands of self-learning and the use of innovative technologies in teaching and learning in health. The aim was to integrate the PBL approach with simulated scenarios, feedback, and self-assessment in an application that is both accessible online and offline from all computing devices including smartphones, tablets, and PCs. Therefore, the application of SavingLife was designed and developed which structures the knowledge of BLS and ACLS in clinical contexts, strengthens motivation to learn, develops clinical reasoning skills, and enhances self-directed learning, anywhere and anytime.

\section{Methodology}

This was a methodological research [24] and technological production of an educational technology named SavingLife. The design and development of the application were completed in 6 months. The development of SavingLife consider the development stages of instructional systems development (ISD) $[25,26]$. The model consists of an action plan, development and implementation of specific teaching scenarios incorporating mechanisms that favor the contextualization of new learning [26].

The methodology involved a process consisted of five steps, which are Analyze, Design, Develop, Implement, and Evaluation $[25,26]$.

- Analyze: This phase consist of gathering information about the target population, the tasks to be completed, theme definition and analysis of available technologies feasible for implementation.

- Design: The design phase consists of writing a learning objective, planning, and production of educational content, preparing the storyboard (screenplay with texts and following screens) and layout design technology (color, font, the arrangement of images and buttons).

- Develop: The development phase involves the creation of the activities that were later implemented. It consists of designing the navigation structure, settings, and programming, generating images, content (Portuguese and English) and animations.

- Implement: This stage consist of the installation of SavingLife files on an online server and submitting to different application stores.

- Evaluate: The final phase, evaluate, ensures the materials achieved the desired goals. This consist of review and testing scenarios of SavingLife with different devices and making necessary adjustments. 


\subsection{Technologies Used}

For SavingLife, the goal was to develop an application which can be accessed through any computing device (mobile, tablets and desktops) with or without internet connectivity. The application was intended to be responsive (adjusted automatically according to the device size) so that it looks good on all devices including small mobile devices, tablets, and desktops.

Two different modules of the application with the same content and design were developed. One was a web-based application accessible from any computing device with an active internet connection. The other was a standalone application (initially for windows platform only) accessible from any windows device. The standalone application can be installed on any windows device in the presence of the Internet and can then later be used offline. Production of standalone application is necessary for offline access (when there is no internet connectivity) while the web-based version is important since it is very difficult to develop the standalone application for all available platforms and operating systems. So developing both these modules were hoped to fill the gap and cover all kind of users.

Hypertext PreProcessor platforms (PHP) and Eclipse were used for the web-based version, available at [27] (username and password: test). PHP is a server-side scripting language designed for web development but can also be used as a general-purpose programming language. In computer programming, Eclipse is an integrated development environment (IDE). It contains workspace and an extensible plug-in system for customizing the environment. Eclipse can be used to develop web and desktop applications.

Visual Studio Community 2015 Edition, a free and rich integrated development environment for creating desktop, web and mobile applications, was used for the development of standalone application (application submitted to Microsoft. Can be downloaded from Windows Store once accepted for inclusion in the Windows store). Visual Studio Community 2015 Edition comes with Xamarin, which is a tool used to share code across multiple platforms and write native Android, iOS, and Windows applications with native user interfaces. Xamarin tool, together with C\# programming language, was used for the development of the standalone application, whose shared code can then be later used to develop native applications for Android and iOS as well.

For the production of two-dimensional graphics, manipulating and retouching photos, MS Paint, GIMP and Adobe Photoshop CS4 were used. For animation, Adobe Flash CS4 (vector graphics program used to create interactive animations) was used.

For managing the database in SavingLife we used MySQL Query Browser and MySQL Administrator tool, to make it possible to insert text for each language, providing greater autonomy for editing content when necessary. MySQL database is selected because it is open source and free, and is the most popular database used worldwide. MySQL Query Browser is a graphical tool made for creating, executing, and optimizing queries in a graphical environment. MySQL Administrator was created to administer a MySQL server.

In order to optimize the data flow in SavingLife, improve interaction with the user, and also facilitate adaptation to different types of mobile devices the following resources were used:

- PHP + MySQL server side: due to easy installation and availability in various hosting environments available.

- Web 2 + Asynchronous JavaScript and XML (AJAX): in order to reduce the amount of data sent from the server to the client.

- Javascript (client side with jQuery 1.5): a cross-browser, open source JavaScript library developed to simplify client-side scripts that interact with HTML, making simpler navigation of the HTML document, creating animations, event handling and development of AJAX applications. It also offers the possibility to create plugins on it.

- WordPress: is a free web software that can be used to create a website, blog, or apps. 


\subsection{Structure}

The seven steps in PBL described by [28] are implemented in SavingLife as shown in Table 1.

Table 1. Structure of SavingLife with respect to PBL.

\begin{tabular}{ll}
\hline PBL & SavingLife \\
\hline Problem Identification & Presentation of learning clinical scenarios \\
Prior Knowledge Exploration & Quizzes pre content and post content \\
Hypothesis \& Action Mechanism & ACLS and BLS scenarios \\
Identify Content for Learning & Get more information about scenarios and Feedback \\
Individual Study (self study) & Scenarios, Simulation, Videos and Knowledge Base \\
Re-Evaluation & Quizzes after content reading (post content) \\
Discussion \& learning evaluation & Quizzes and Feedback \\
\hline
\end{tabular}

The navigation structure of the SavingLife is shown in Figure 1. It must be noted that users can access the clinical scenario, simulations, and quizzes in a non-linear way, without any specific order to navigate the SavingLife leaving students free to establish the construction of knowledge from their past experiences.

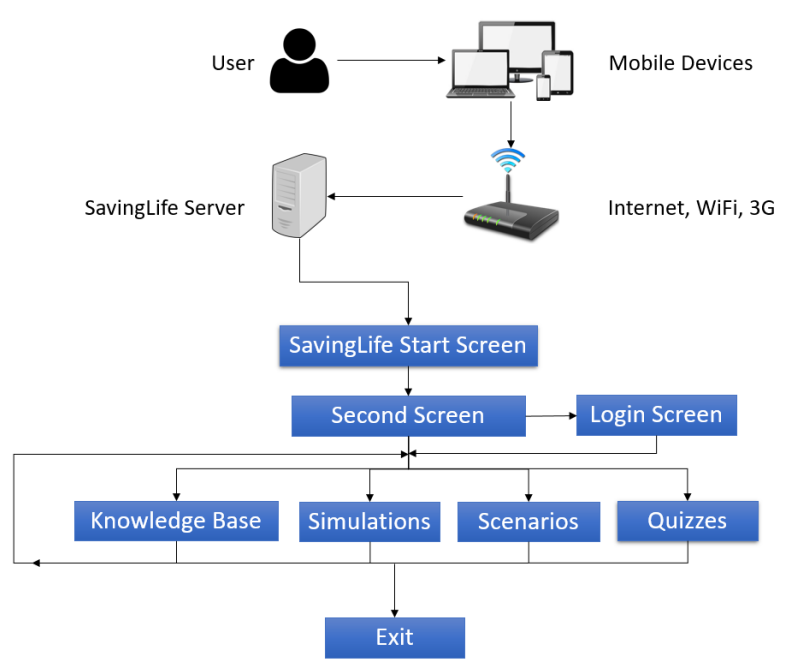

Figure 1. Navigation structure of SavingLife.

The contents in SavingLife have been organized in Scenarios, Simulations, Algorithms, Knowledgebase, Quizzes, and Videos as shown in Figure 2.

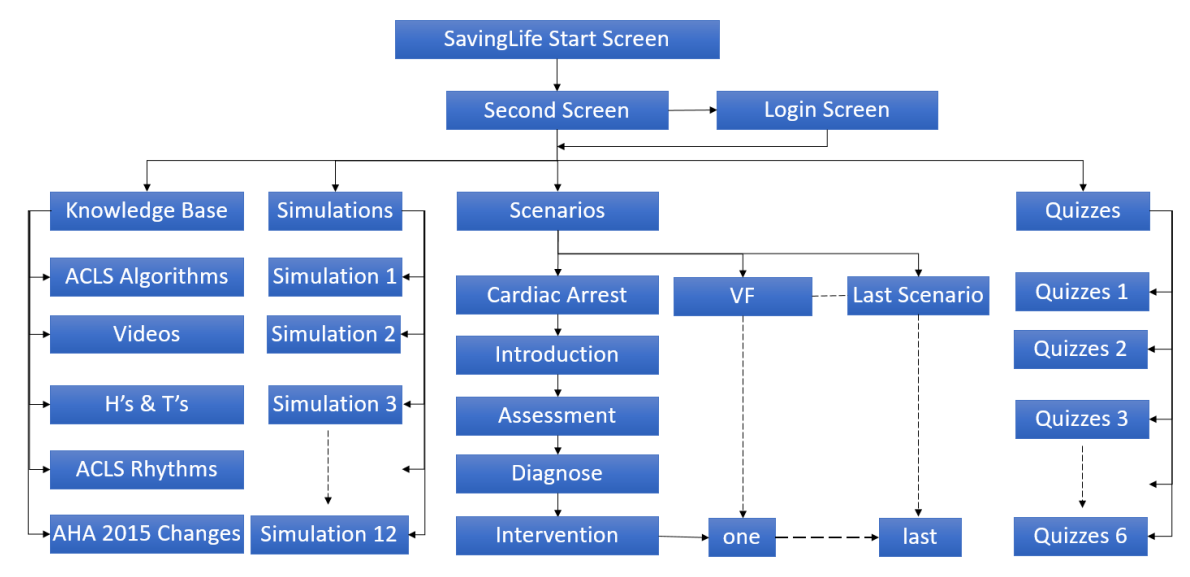

Figure 2. Content structure of SavingLife. 


\section{Results}

An educational instructional technology (SavingLife) based on the approach of problem-based learning, was developed for enhancing the learning of basic and advanced cardiovascular life support. Two different modules of SavingLife were produced. One is a web-based application accessible through any computing device which has a web browser and an active internet connection. The web-based version is available at [27]. The other is a standalone application (initially developed for windows platform only) accessible through any windows device. The standalone application can be installed on any windows device in the presence of the Internet and can then later be used offline.

Standalone applications are useful for offline access (when there is no internet connectivity) while web-based applications are effective for online access since it is very difficult to develop a standalone application for all available platforms and operating systems. The design, architecture, look and feel of both versions is essentially the same on all devices including smartphones, tablets, laptops, and desktops.

The application interface is responsive and adjusts automatically according to the screen size of the device (see Figures 3 and 4 for comparisons). All the content of SavingLife is based on the updated 2015 American Heart Association (AHA) guidelines for BLS and ACLS. The content of SavingLife is presented in two languages (English and Portuguese). The content language of SavingLife is automatically selected according to the default language of the operating system of the device from which the application is accessed. However, preferred language can be manually switched anytime from within the application menu.

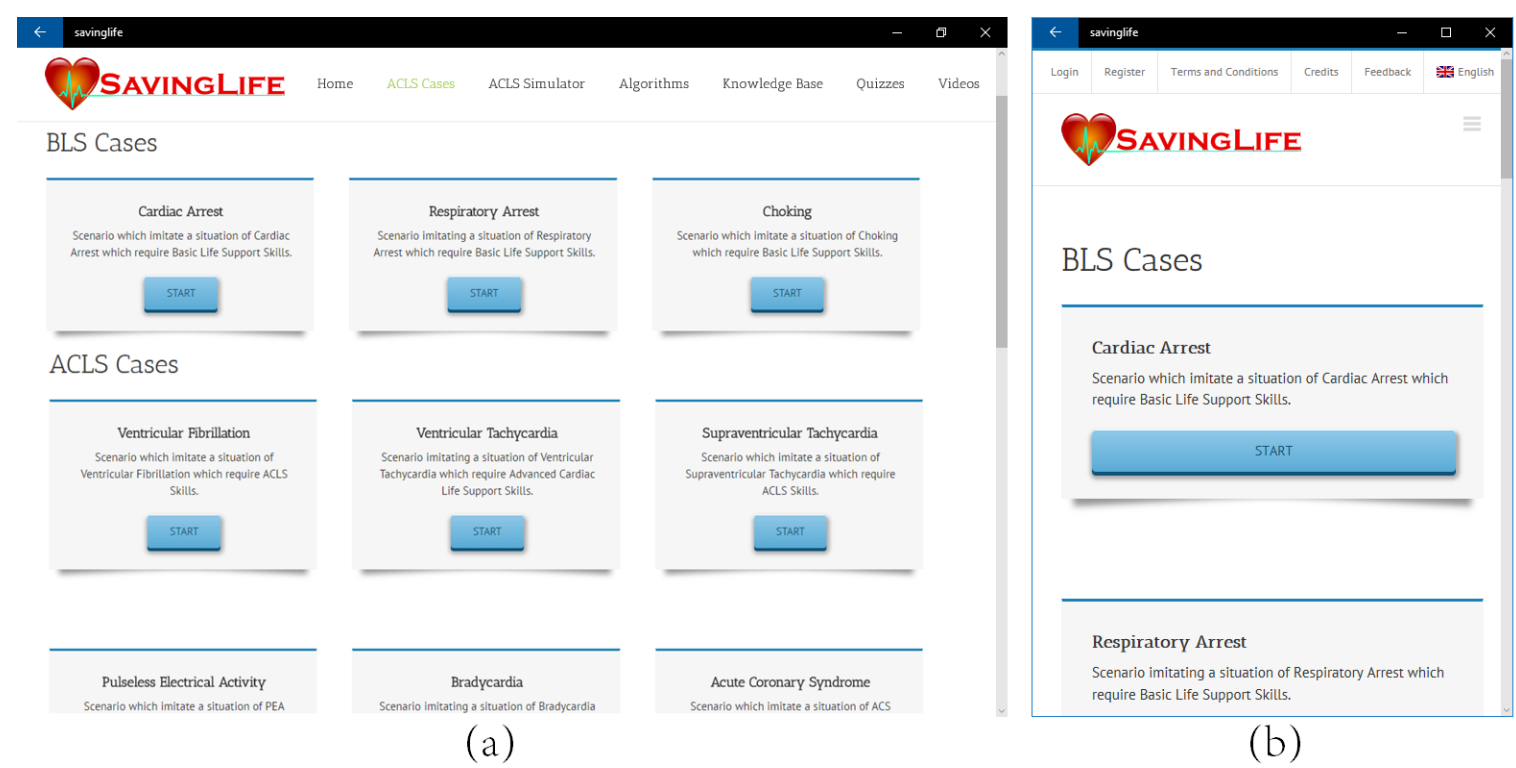

Figure 3. Scenarios main screen on (a) large screen, (b) small screen. 


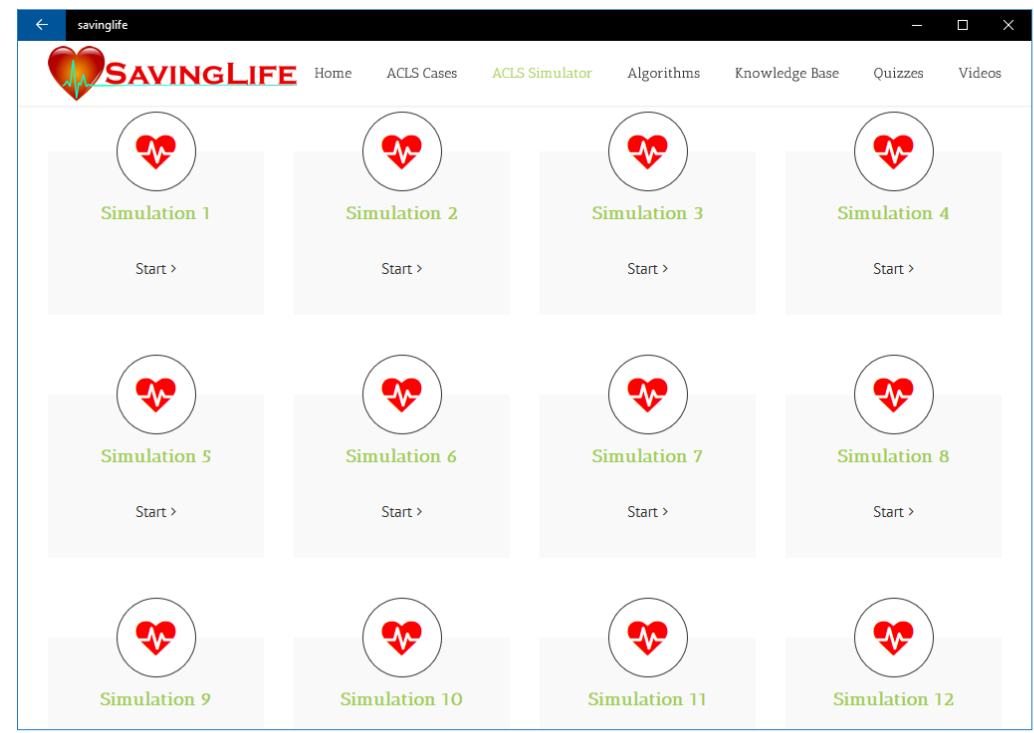

(a)

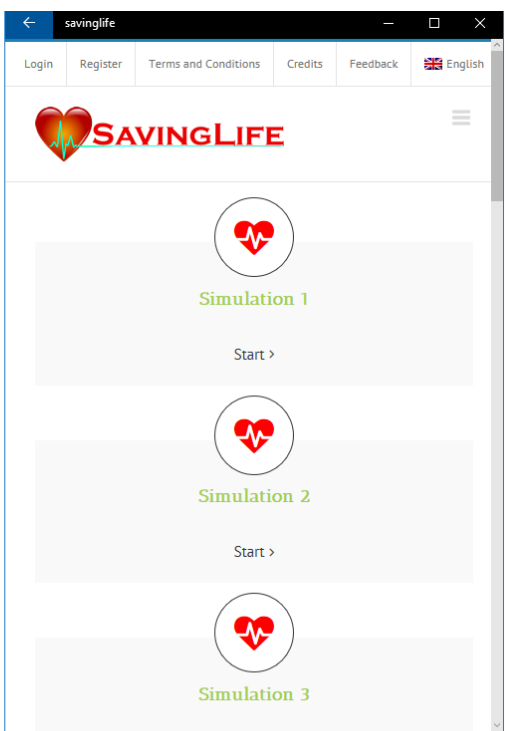

(b)

Figure 4. Simulations main screen on (a) large screen, (b) small screen.

The first screen (Figure 5a) presents the name, logo, and authorship of SavingLife along with the institution of origin and year of production. The second screen (Figure $5 b$ ) shows a brief introduction to the SavingLife application. The third screen reveals a login form (Figure 6) if the user is not already logged in or the scenario screen (Figure 3) if the user is already logged in.

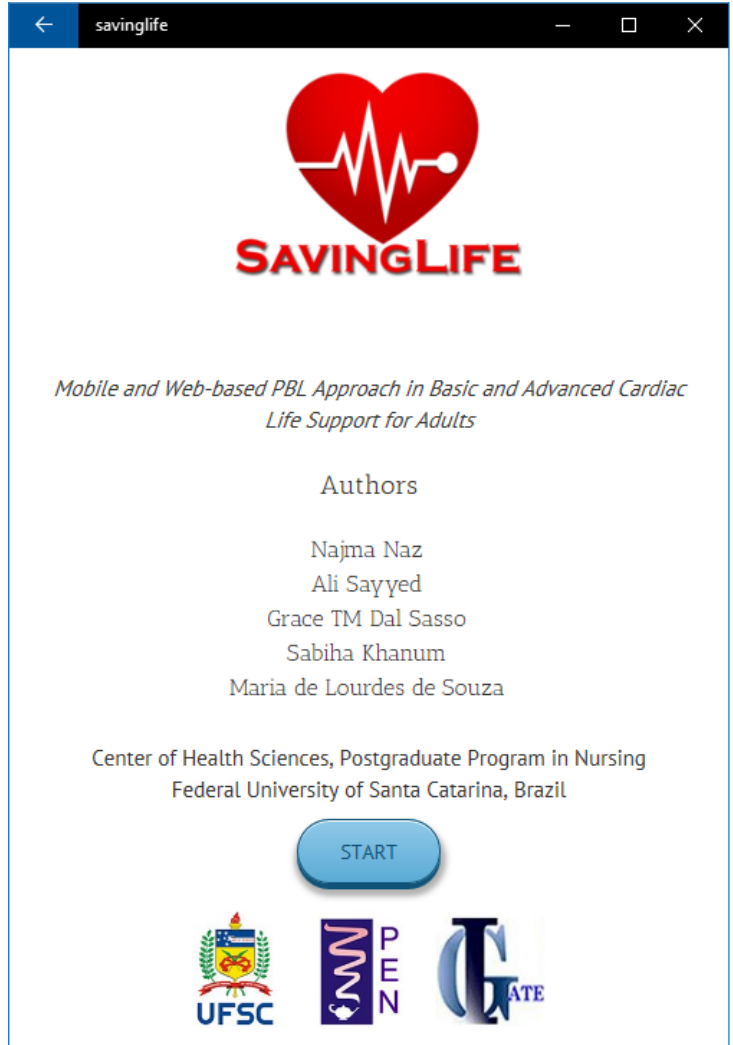

(a)

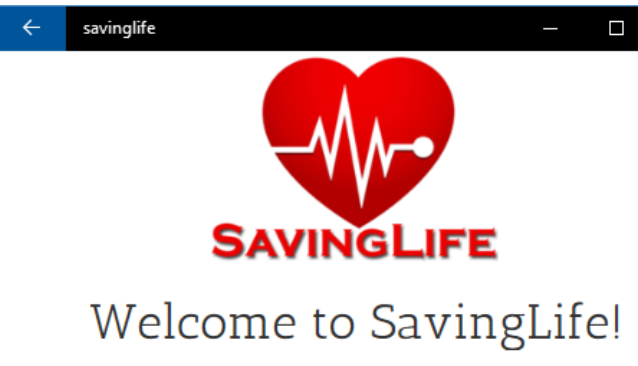

This is a mobile and web based application built on the concept of Problem Based Learning. Here, you can learn and improve your basic and advanced cardiac life support skills for adult patients using different real life scenarios. This online learning experience will give you the confidence that you need in emergency situations.

The information and scenarios described in the SavingLife is fictitious, but it simulates the real life situations. To meet the objectives, you may start by assessing the patient. After assessment, determine the most appropriate diagnosis and apply the necessary interventions. Click "Continue" to start learning.

\section{CONTINUE}

(b)

Figure 5. (a). First screen of SavingLife; (b). Second screen of SavingLife. 


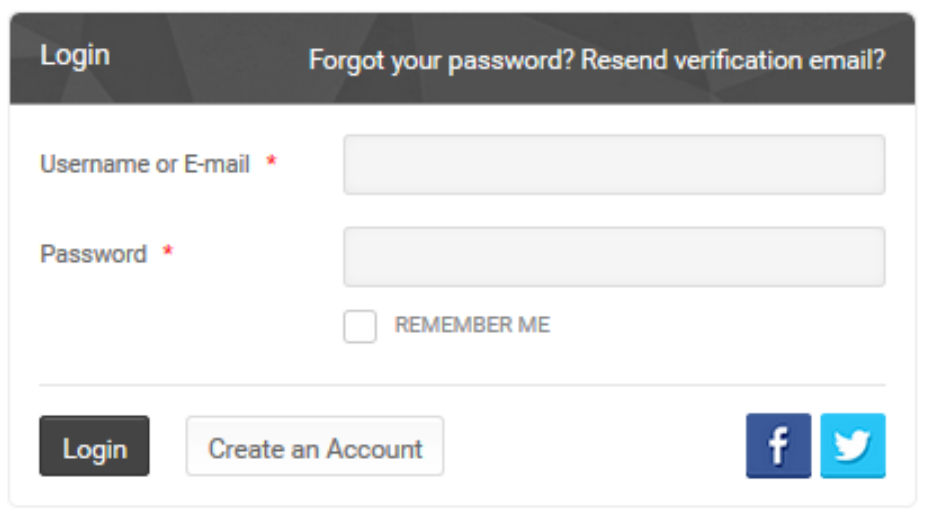

Figure 6. Login screen of SavingLife.

The contents in SavingLife have been organized in a way to offer students the options to improve their learning and perform a self-evaluation. Six learning nodes namely Scenarios, Simulator, Algorithms, Knowledgebase, Quizzes, and Videos, are created. It must be noted that the student can select any node from the main menu without a mandatory order (Non-linear model) leaving learners free to improve their knowledge from their experiences.

\subsection{Cases / Scenarios}

After successfully logging in, the user is directed to the selection screen of clinical scenarios Contents are organized in 10 different cases which representing the scenarios of real-life situations covering BLS and ACLS guidelines according to the AHA 2015. These cases include respiratory arrest, cardiac arrest, choking, ventricular fibrillation, pulseless electrical activity, pulseless ventricular tachycardia, supraventricular tachycardia, bradycardia, acute coronary syndrome, and suspected stroke.

Each case consists of one fictitious problem situation of cardiac arrest, arrhythmias or other special circumstances. Students can try to solve the problem through step by step guideline of AHA.

Each clinical scenario presents a patient with necessary details. When a user clicks to start learning a case, first they are presented with a brief introduction and case description as shown in Figure 7. Then the user moves on to the next screen for necessary assessment, as shown in Figure 8.

Assessment of the patient can be performed by virtual communication with the victim through different options available on the assessment screen. After the assessment, the student makes a hypothesis and diagnose the patient using the option available on the diagnose screen (Figure 9). After successful diagnose, the user immediately begins intervention and re-evaluation as shown in Figure 10. The cycle of intervention and re-evaluation continues until the victim is stabilized. In this way, the students continue to use the essential steps of assessment, diagnose, intervention and evaluation while improving their knowledge and skill. The students have different options to decide on the actions to be taken during different scenarios considering real situations of nursing practice.

After each (re)evaluation, the select the right option from the available choices. On selecting the right option the scenario moves forward. Depending on the condition the victim is then reevaluated and next intervention is decided. On selecting a wrong option, the application let the student try again for the right option until the right option is selected.

Backward button, when a scenario is in progress, is not given intentionally since the scenarios are based on the approach of PBL. The aim was to present the scenario as close to a real-life situation as possible. Such a situation only moves forward and the student has to focus on the current evaluation and decide on the next interventions. However, the student can always restart a scenario whenever required. 


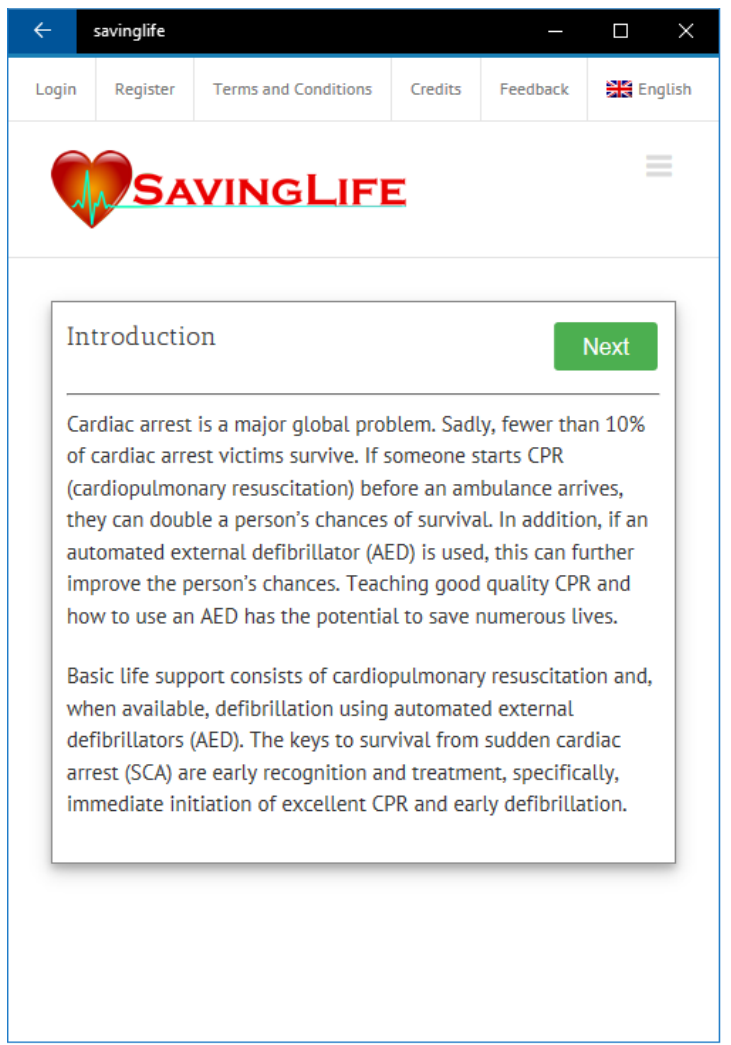

(a)

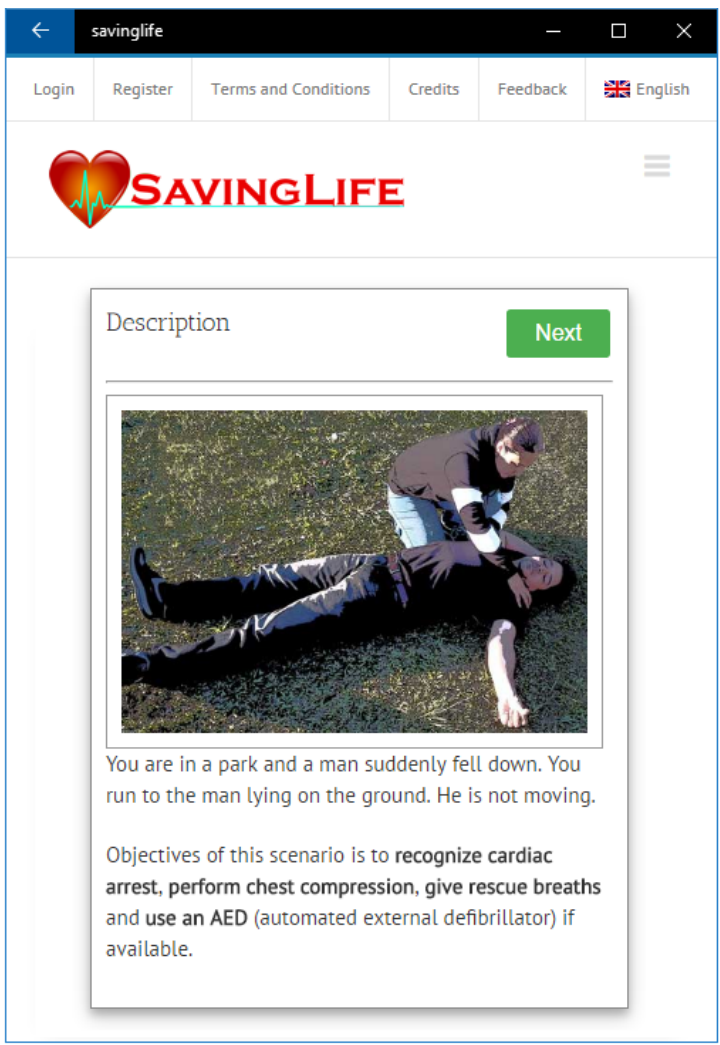

(b)

Figure 7. (a) Introduction of a scenario; (b) Description of a scenario.

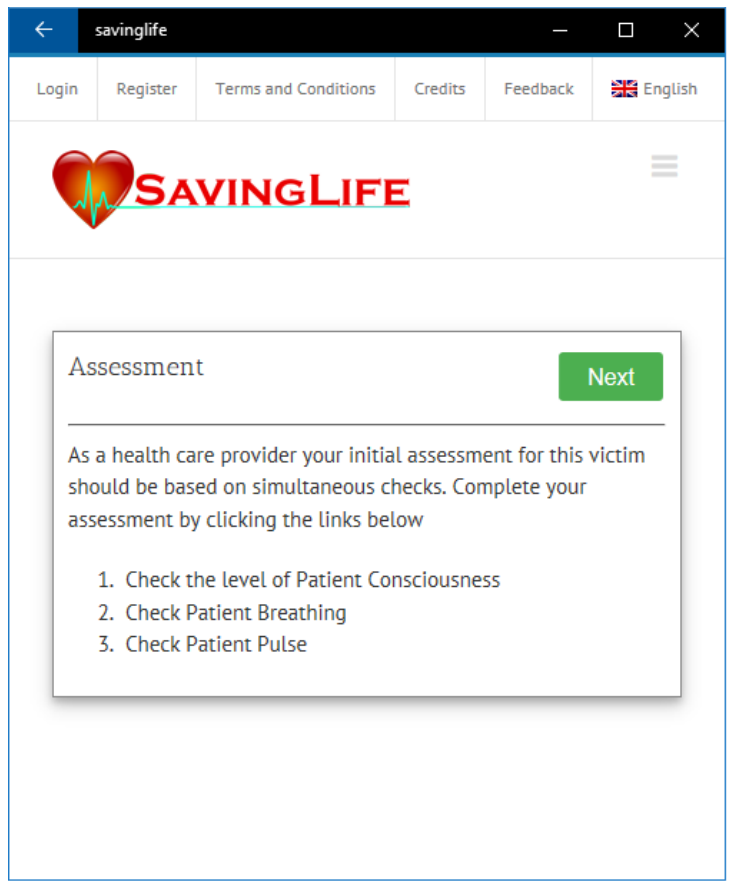

(a)

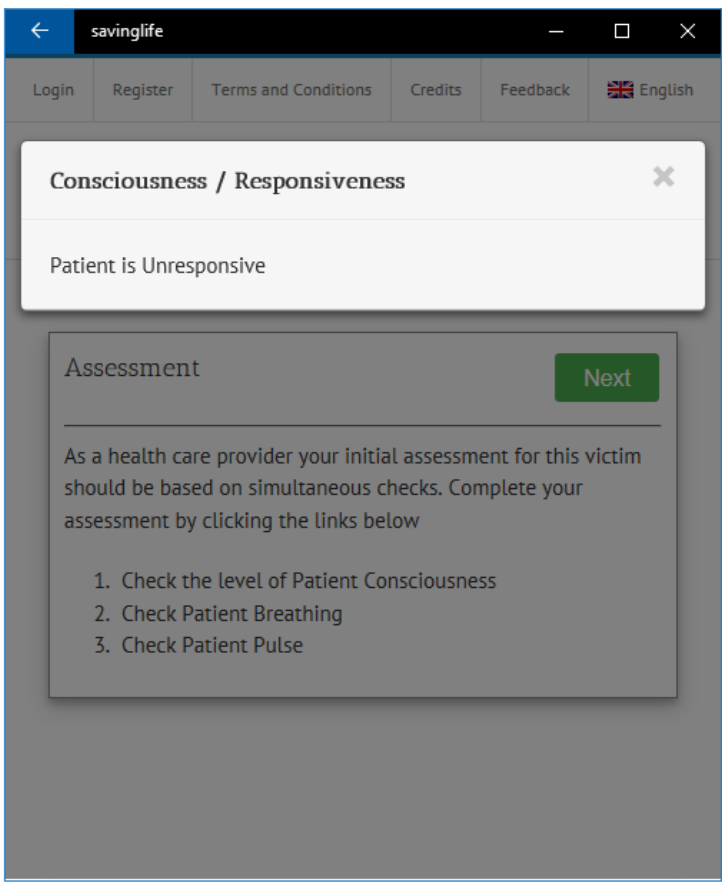

(b)

Figure 8. (a) Assessment screen (b) Simulated victim response when first option is clicked. 


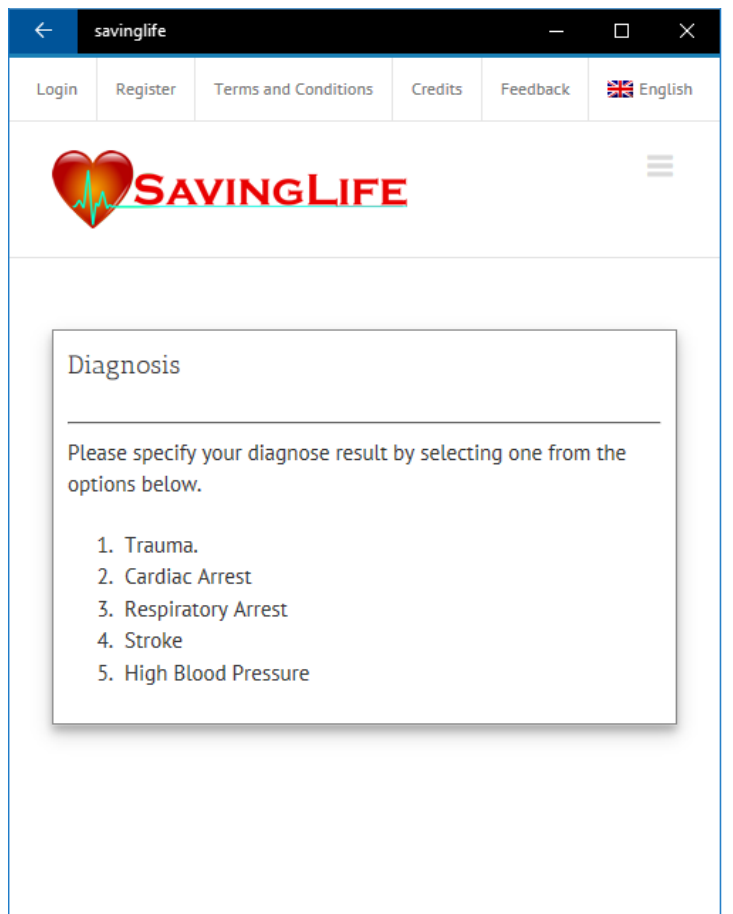

(a)

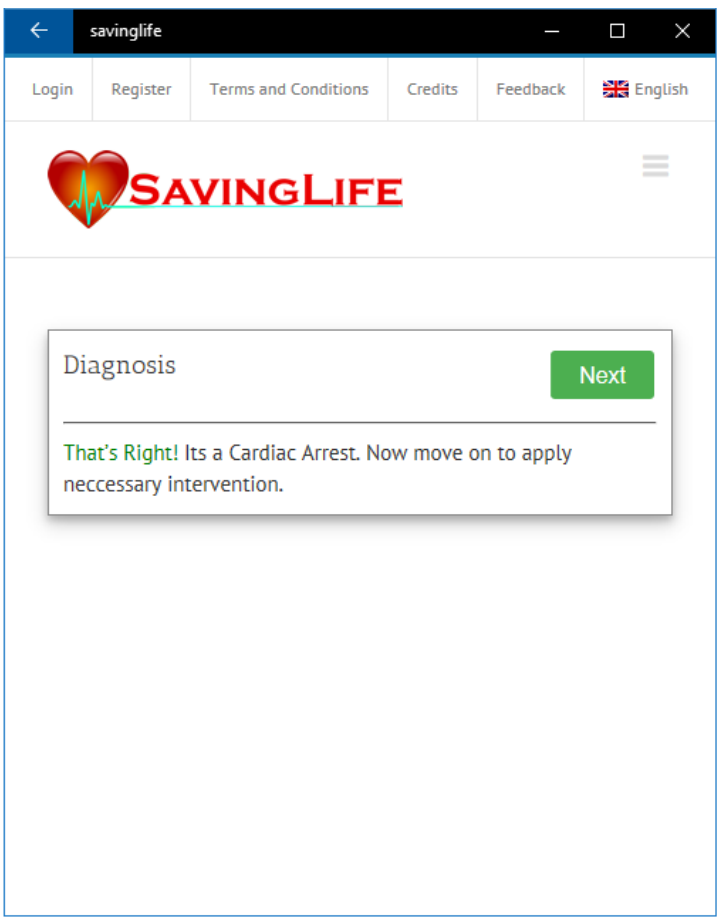

(b)

Figure 9. (a) Diagnosis screen (b) Response of the system when the right option (the 2nd) is clicked.

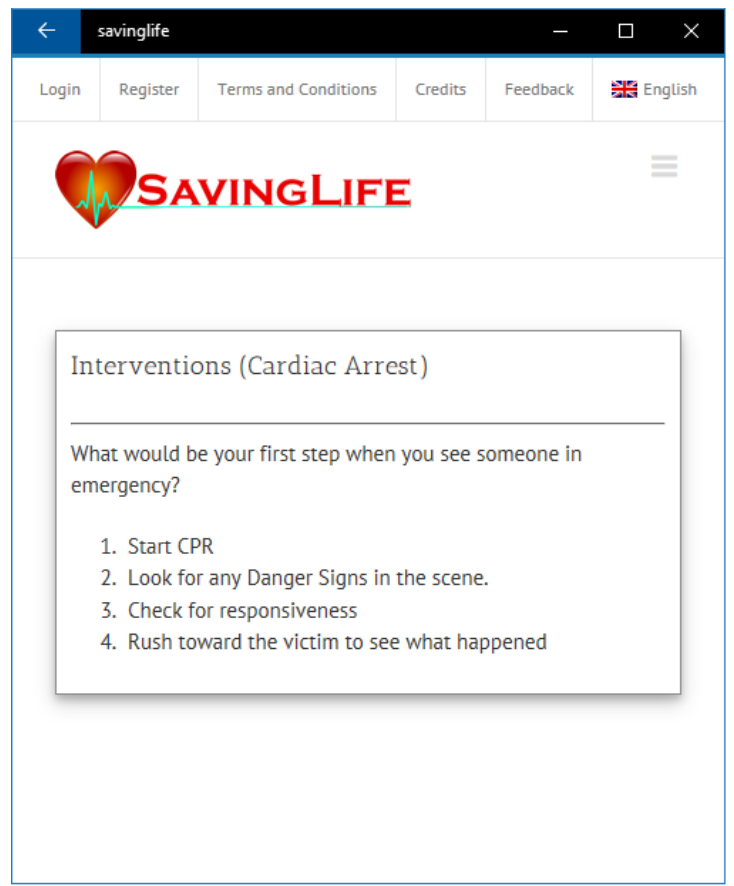

(a)

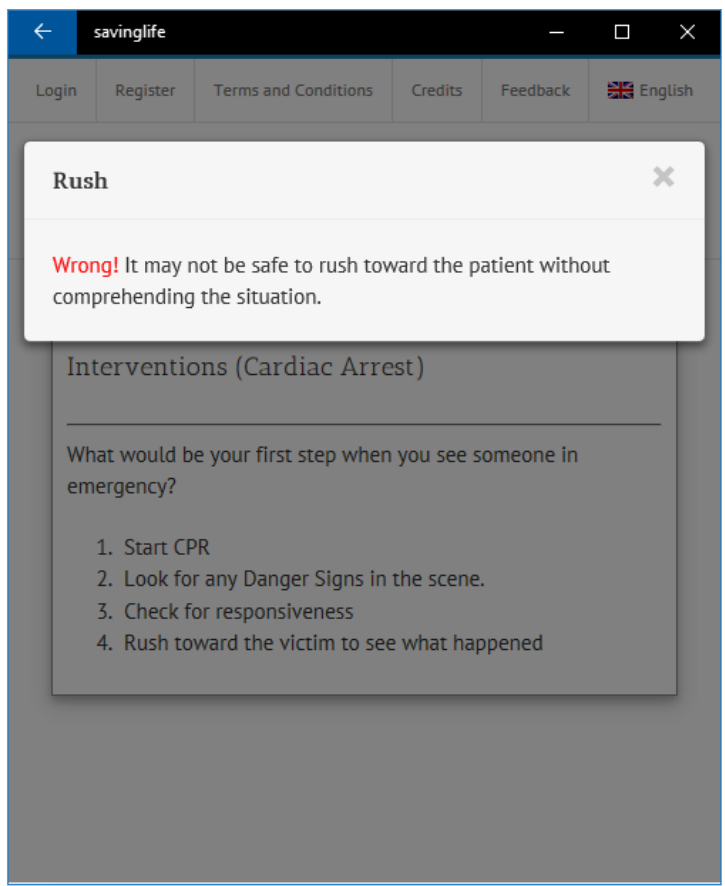

(b)

Figure 10. (a) Interventions screen (b) Response of the system when a wrong option (the 4rth) is clicked.

\subsection{Simulations}

The Simulations section (see Figure 4) presents total twelve real-life situations. The scenarios in this section are almost similar to the ones in the Scenarios section but are more real and at an advanced level. 
The purpose of simulations is to enable students to learn and handle all the possible emergencies occurred in Cardiac Arrest or in ACLS in the intensive coronary care unit. Unlike the situations in Scenario section, in simulation students are presented only the signs, symptoms and rhythm (see Figure 11) from which they identify the case and continue on with the necessary interventions. In simulations, the patient condition changes from one algorithm to another algorithm, thus mimicking a closer real-life patient.

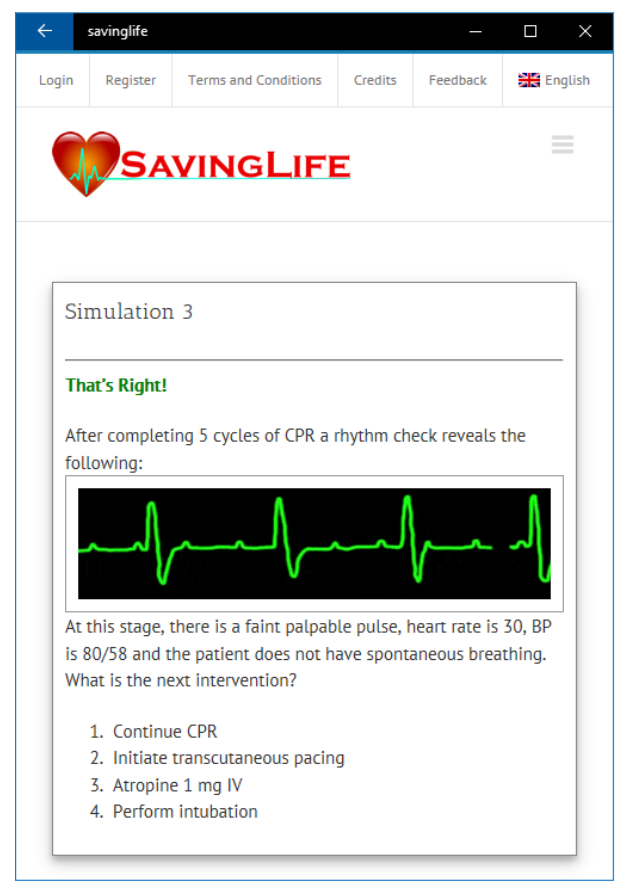

(a)

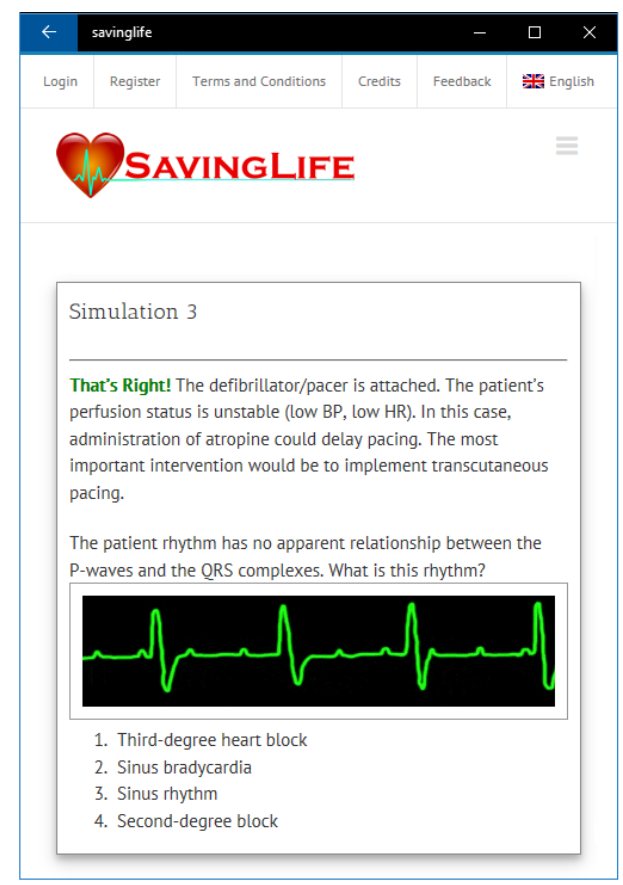

(b)

Figure 11. (a) A screen from simulation 3; (b) Response of the system when the right option (the 2nd) is clicked.

\subsection{Algorithms}

Algorithms are one of the most important components in effective learning of BLS and ACLS. Every emergency health care provider and nurse can save a life if they are familiar with ACLS algorithms. Algorithms are step by step procedures to solve a particular problem. Six different algorithms, adopted from the updated 2015 AHA guidelines [29], available in SavingLife are:

- $\quad$ BLS Primary Survey (Figure 12a)

- Adult Cardiac Arrest (for VF, pVT, PEA, and Asystole)

- Adult Ventricular Tachycardia (Figure 12b)

- Adult Bradycardia

- Acute Coronary Syndrome

- Suspected Stroke 


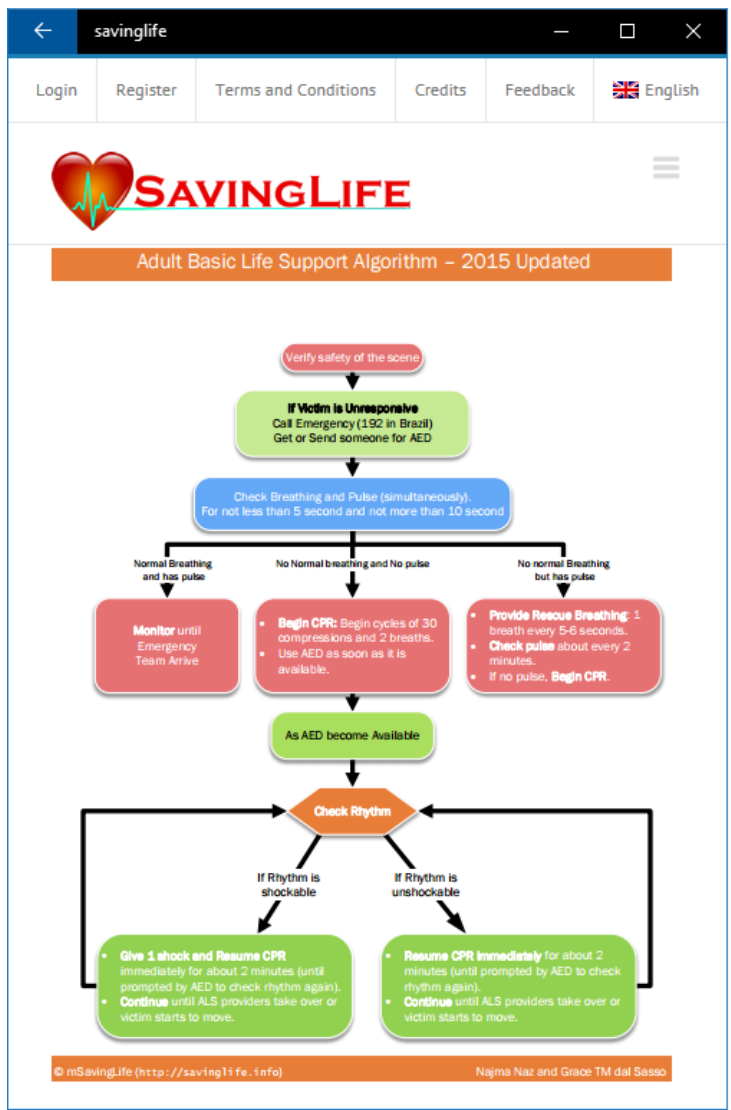

(a)

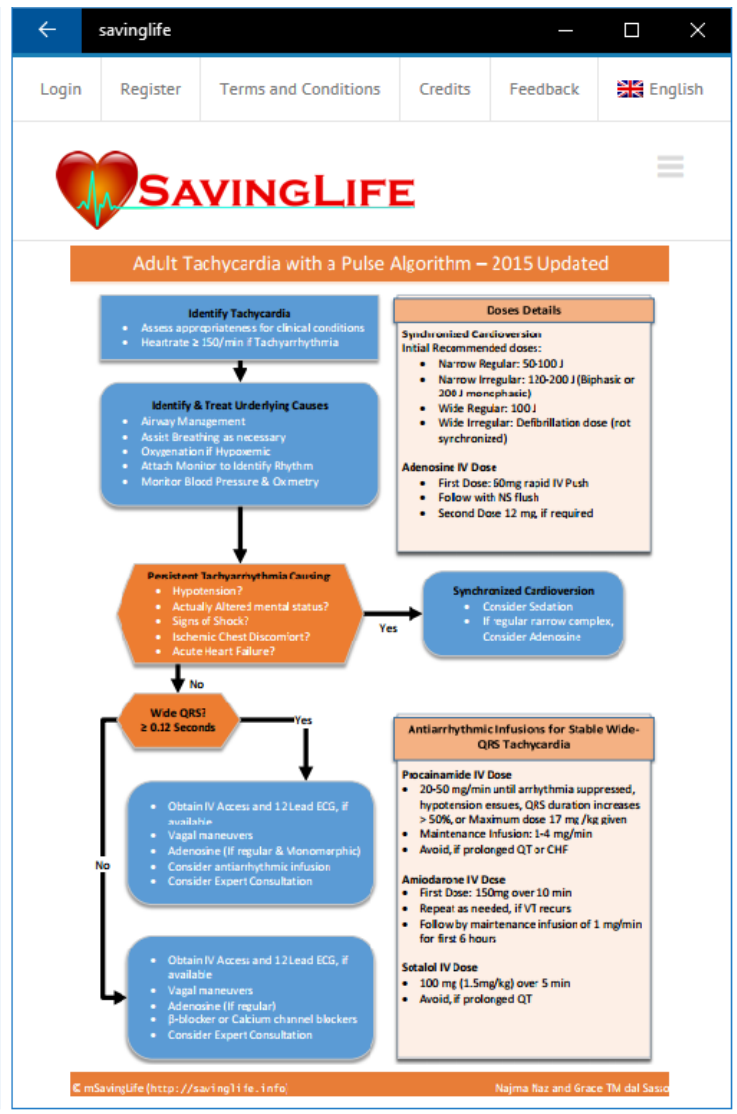

(b)

Figure 12. (a) Adult basic life support algorithm; (b) Adult tachycardia with a pulse algorithm.

\subsection{Knowledge Base}

The knowledge base is designed to help students become familiar with the prerequisite knowledge and improve their learning. The knowledge base includes the H's and T's of ACLS, 2015-2020 ACLS Changes Updates by AHA and ACLS Rhythms Identification and Interpretation as shown in Figure 13a.

H's and T's: While all algorithms in SavingLife contain step-by-step, definitive treatment interventions. It often requires professionals to identify and address the causal factor associated with cardiopulmonary arrests. $\mathrm{H}$ and $\mathrm{T}$ are most commonly associated with PEA but it also helps in finding the underlying causes for any of the arrhythmias associated with ACLS [9]. H's and T's in SavingLife help prepare you for any ACLS scenario.

Rhythm identification and recognition: Adults BLS and ACLS require that health care providers should be able to recognize and interpret several basic cardiac rhythms from normal sinus rhythm to Asystole. This section describes 12 of the most commonly encountered ECG animated rhythms.

2015-2020 AHA Changes: American Heart Association released their new updated guidelines for BLS and ACLS in October 2015. These guidelines will be useful through 2020. They made some minor changes to both the BLS and ACLS Guidelines. The article content was developed from information released by the American Heart Association [9]. 


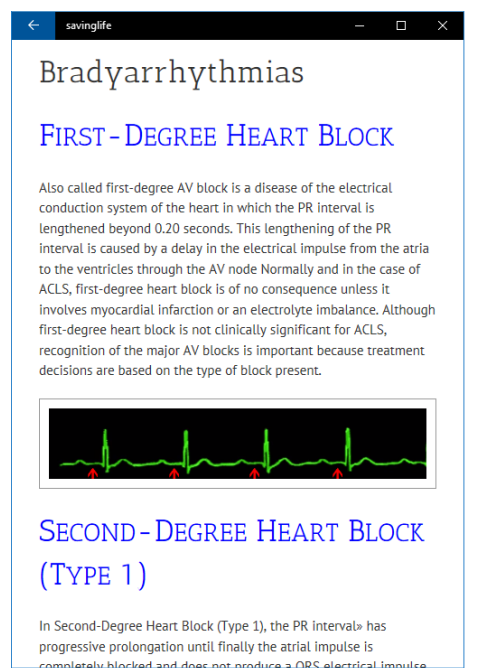

(a)

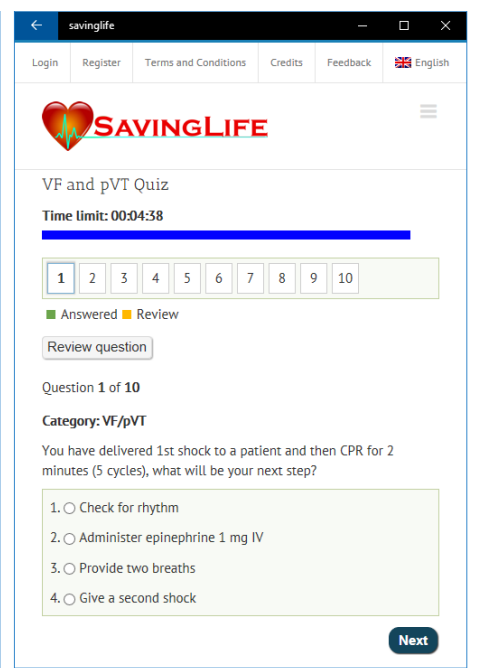

(b)

Figure 13. (a) Learning rhythms interpretations and identifications. (b) Quiz for self evaluation.

\subsection{Quizzes}

Self-assessment had an important role in active learning [30]. The main purpose of quizzes, a tool for self-evaluation and self-assessment in SavingLife, is to enable students to evaluate their pre and post-learning. The quizzes section consists of BLS and ACLS practice test (four cardiac arrest cases), bradycardia, and tachycardia. A variety of questions covering all aspect of different cases are presented. This section tests the knowledge of the systematic approach of students to treat acutely ill cardiac arrest patients, with the return of spontaneous circulation (ROSC) being the ultimate goal. A sample test is shown in Figure 13b. A timer (30 s for each question) is used for each quiz in order to improve the power of quick decisions in a situation where time is of extreme value. Students can see their progress and scores (see Figure 14a) along with the leaderboard and can identify their mistakes and improve the weak point by reviewing their answers (see Figure 14b). Each time the student starts a quiz, all the questions and answer shuffles and their order and position changes so that the student learning ability is effectively evaluated. In addition to the quizzes, the scenarios and simulation also offer a tool for students to improve as well as evaluate their knowledge about BLS and ACLS.

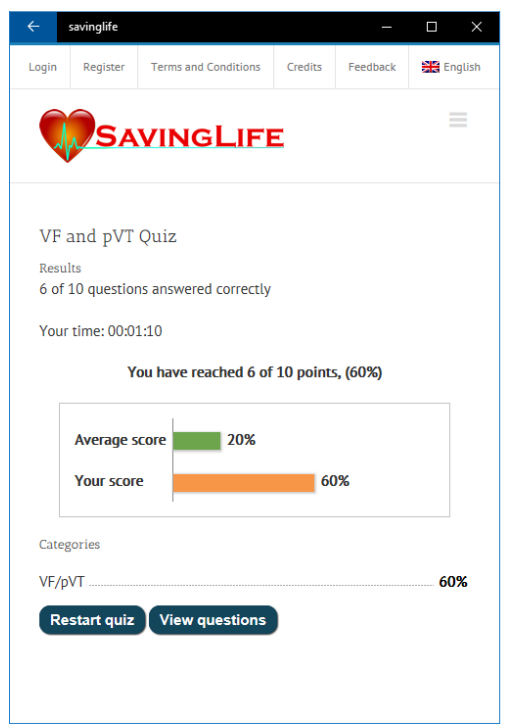

(a)

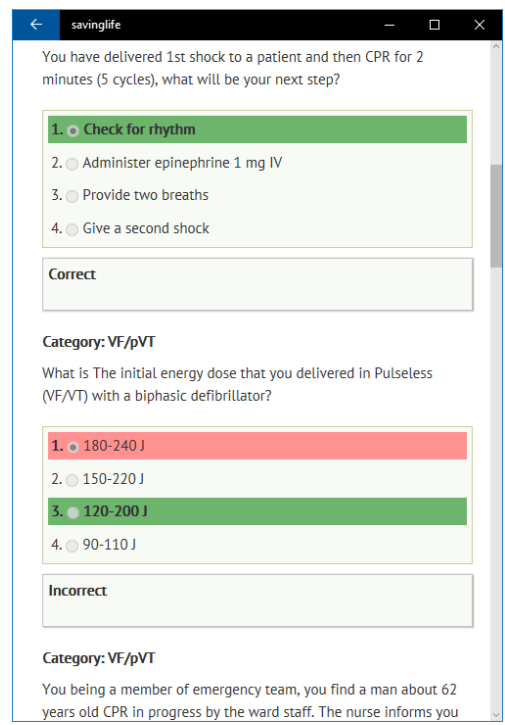

(b)

Figure 14. (a) Quiz results and Leaderboard; (b) Quiz review. 


\subsection{Videos}

Three videos regarding BLS, VF/pVT, and Asystole in order to improve the confidence of students while practicing BLS and ACLS skills. More videos covering different details of all BLS and ACLS aspects are intended to be provided as an additional media for learning.

\subsection{Feedback}

Although self-learning methods through computer applications are considered to be useful to learn BLS and ACLS, it should be noted that the instructor's feedback for answering student questions related to the emergency conditions is important. In PBL, it is also important that each student have an opportunity to reflect on the process of learning that has taken place. This includes reviewing the learning achievements and listing ideas, improvements, and deficiencies in the learning process so that it can be consolidated for future application [28]. For this, a feedback option is available where a student can establish asynchronous communication with the administrator and teacher for additional help and guidance.

\section{Limitations}

The current educational technology opens the possibility of mass public training via the Internet. However, it would be important to ensure that participants receive sufficient incentive or motivation to use the online program or download it to their mobile devices.

Furthermore, this method could be more useful if each student in web-based instruction group is provided with Mini CPR Kit to provide them with a self-learning CPR tool. It should not be seen as replacing other methods of training, and practice on a manikin should be encouraged when circumstances permit.

\section{Conclusions}

The rapid growth and wide availability of information and communication technologies are paving the way for innovative, motivating and flexible learning methods. ICT-based educational technologies can play a vital role in facilitating low-cost effective educational services not restricted to space or time.

SavingLife, an ICT-based educational technology was designed and developed incorporating problem-based learning approach for training nurses and other health students in BLS and ACLS skills. SavingLife meet both the demands of self-learning and the use of innovative and widely accessible technologies in teaching and learning in health. It is believed that SavingLife offers an interactive and flexible tool that strengthens the motivation to learn and develops clinical reasoning skills. SavingLife can contribute to the student's learning and helps to fill the gaps in the assessment and management of cardiac arrest, stroke and other life-threatening medical emergencies, in a safe and ethical manner.

Author Contributions: N.N., A.S., and G.T.M.D.S. conceived the idea of the SavingLife and did the initial literature review. N.N. and S.K. prepared the manuscript. N.N., G.T.M.D.S., and M.d.L.d.S. designed and prepared the block diagrams and application screens. A.S. did all the programming and coding of the application. All authors edited the manuscript and approved the final version to be published.

Acknowledgments: We wish to thank CNPq (Conselho Nacional de Desenvolvimento Científico e Tecnológico Brazil) and CAPES (Coordenação de Aperfeiçoamento de Pessoal de Nível Superior Brazil) for their financial assistance.

Conflicts of Interest: The authors declare no conflict of interest. 


\section{References}

1. Mark, S.; Lauren, L.; Berkow, C.; Peter, J.; Henry, K.; Halperin, R.; Erik, P.; Vivek, H.; Moitra, K.; Robert, W.; et al. Adult Advanced Cardiovascular Life Support 2015 American Heart Association Guidelines Update for Cardiopulmonary Resuscitation and Emergency Cardiovascular Care. Circulation 2015, 132, 1161-1261.

2. Otero, R.M. Cardiac Arrest Management. In Evidence-Based Critical Care; Springer: Cham, Switzerland, 2017; pp. 3-11.

3. Roger, V.L.; Go, A.S.; Lloyd-Jones, D.; Benjamin, E.; Berry, J.; Borden, W.; Bravata, D.; Dai, S.; Ford, E.; Fox, C.; et al. American heart association statistics committee and stroke statistics subcommittee. Heart disease and stroke statistics 2011 update: A report from the American Heart Association. Circulation 2011, 123, e18-e209.

4. WHO. Cardiovascular Diseases; WHO: Geneva, Switzerland, 2016.

5. Nichol, G.; Thomas, E.; Callaway, C.W.; Hedges, J.; Powell, J.L.; Aufderheide, T.P.; Rea, T.; Lowe, R.; Brown, T.; Dreyer, J.; et al. Regional variation in out-of-hospital cardiac arrest incidence and outcome. JAMA 2008, 300, 1423-1431.

6. Nadkarni, V.M.; Larkin, G.L.; Peberdy, M.A.; Carey, S.M.; Kaye, W.; Mancini, M.E.; Nichol, G.; Lane-Truitt, T.; Potts, J.; Ornato, J.P.; et al. First documented rhythm and clinical outcome from in-hospital cardiac arrest among children and adults. JAMA 2006, 295, 50-57.

7. Mozaffarian, D.; Benjamin, E.J.; Go, A.S.; Arnett, D.K.; Blaha, M.J.; Cushman, M.; Das, S.R.; de Ferranti, S.; Després, J.P.; Fullerton, H.J.; et al. Executive Summary: Heart Disease and Stroke Statistics-2016 Update: A Report From the American Heart Association. Circulation 2016, 133, 447.

8. American Heart Association. 2010 American Heart Association Guidelines for Cardiopulmonary Resuscitation and Emergency Cardiovascular Care; American Heart Association: Dallas, TX, USA, 2010.

9. Link, M.S.; Berkow, L.C.; Kudenchuk, P.J.; Halperin, H.R.; Hess, E.P.; Moitra, V.K.; Neumar, R.W.; O’Neil, B.J.; Paxton, J.H.; Silvers, S.M.; et al. Part 7: Adult advanced cardiovascular life support 2015 american heart association guidelines update for cardiopulmonary resuscitation and emergency cardiovascular care. Circulation 2015, 132, S444-S464.

10. Gombotz, H.; Weh, B.; Mitterndorfer, W.; Rehak, P. In-hospital cardiac resuscitation outside the ICU by nursing staff equipped with automated external defibrillators: The first 500 cases. Resuscitation 2006, 70, 416-422.

11. Sardo, P.M.G.; Dal Sasso, G.T.M. Problem-based learning in cardiopulmonary resuscitation: Basic life support. Rev. Esc. Enferm. USP 2008, 42, 784-792.

12. Ozturk, C.; Muslu, G.K.; Dicle, A. A comparison of problem-based and traditional education on nursing students' critical thinking dispositions. Nurse Educ. Today 2008, 28, 627-632.

13. DeVita, M. Organizational factors affect human resuscitation: The role of simulation in resuscitation research. Crit. Care Med. 2005, 33, 1150-1151.

14. Hosseini, S.K.; Ghalamkari, M.; Yousefshahi, F.; Mireskandari, S.M.; Hamami, M.R. Advanced Cardiac Life Support Training by Problem-Based Method: Effect on the Trainee's Skills, Knowledge and Evaluation of Trainers. J. Tehran Univ. Heart Cent. 2013, 8, 187.

15. The World Bank. Mobile Cellular Subscriptions; The World Bank: Washington, DC, USA, 2016.

16. Statista Inc. The Statistics Portal-Mobile Phone Users Worldwide; Statista Inc.: Hamburg, Germany, 2016.

17. Central Intelligence Agency. The World Factbook; Central Intelligence Agency: McLean, VA, USA, 2016.

18. West, D. How mobile devices are transforming healthcare. Issues Technol. Innov. 2012, 18, 1-11.

19. Speciale, A.M.; Freytsis, M. mHealth for midwives: A call to action. J. Midwifery Women's Health 2013, $58,76-82$.

20. Boulos, M.N.; Maramba, I.; Wheeler, S. Wikis, blogs and podcasts: A new generation of Web-based tools for virtual collaborative clinical practice and education. BMC Med. Educ. 2006, 6, 41.

21. de Vries, W.; Handley, A.J. A web-based micro-simulation program for self-learning BLS skills and the use of an AED: Can laypeople train themselves without a manikin? Resuscitation 2007, 75, 491-498.

22. Saraç, L.; Ok, A. The effects of different instructional methods on students' acquisition and retention of cardiopulmonary resuscitation skills. Resuscitation 2010, 81, 555-561.

23. Kardong-Edgren, S.E.; Oermann, M.H.; Odom-Maryon, T.; Ha, Y. Comparison of two instructional modalities for nursing student CPR skill acquisition. Resuscitation 2010, 81, 1019-1024. 
24. Polit, D.F.; Beck, C.T. Nursing Research: Principles and Methods; Lippincott Williams \& Wilkins: Philadelphia, PA, USA, 2004.

25. Dick, W.; Carey, L.; Carey, J.O. The Systematic Design of Instruction; Longman: New York, NY, USA, 2001; Volume 5.

26. Filatro, A.; Piconez, S.C.B. Design Instrucional Contextualizado; Senac: São Paulo, Brazil, 2004.

27. Naz, N.; Sayyed, A.; Dal Sasso, G.; Khanum, S.; de Souza, M.d.L. SavingLife, a Mobile and Web-based PBL Approach in Basic and Advanced Cardiac Life Support for Adults. Available online: http:/ / savinglife.net/ (accessed on 1 January 2018).

28. Walsh, A. The Tutor in Problem-Based Learning: A Novice's Guide; McMaster University: Hamilton, ON, Canada, 2005.

29. American Heart Association. Highlights of the 2015 American Heart Association Guidelines Update for CPR and ECC; American Heart Association: Dallas, TX, USA, 2016.

30. Boud, D. Enhancing Learning through Self-Assessment; Routledge: London, UK, 2013.

(C) 2018 by the authors. Licensee MDPI, Basel, Switzerland. This article is an open access article distributed under the terms and conditions of the Creative Commons Attribution (CC BY) license (http:/ / creativecommons.org/licenses/by/4.0/). 